\title{
Estrategia restrictiva transfusional en cirugía ortopédica
}

\author{
Marcos Pintos ${ }^{*}$, Verónica Ventura†, Maximiliano Tarallo \\ Daniel Rienzis, Jorge Decaro", Mario Cancela** \\ Asociación Española, Montevideo, Uruguay
}

\section{Resumen}

Para pacientes que sufren artrosis avanzada o en algunos casos de fractura de cadera, la solución quirúrgica que se propone es la cirugía ortopédica mayor. Alguno de estos pacientes requerirá transfusión de sangre o hemocomponentes, 0 ambos, antes o después de la intervención.

El abordaje de la anemia en el paciente quirúrgico puede hacerse de manera liberal, sujeto a la indicación de cada cirujano, o en el contexto de un protocolo restrictivo de reposición. Con este último el beneficio en el ahorro de recursos se acompaña además de un menor número de complicaciones relacionadas con la administración de sangre alogénica.

El objetivo específico de nuestro trabajo fue comparar la estrategia liberal con la terapia restrictiva transfusional.

Ingresaron en el estudio 498 pacientes; de estos, 261 (52,4\%) en el año 2014 sometidos a terapia liberal y $237(47,6 \%)$ en el año 2015 a quienes se aplicó el protocolo de terapia restrictiva transfusional. Se transfundieron menos individuos y se indicaron $55 \%$ menos volúmenes de sangre con la estrategia restrictiva.

Se presentaron diez casos de reacción transfusional, todas de tipo febril, ocho en 2014 y dos en 2015. Con respecto a la evolución funcional no se comprobó diferencia entre ambos grupos.

En nuestra experiencia y de acuerdo a la literatura consultada, la terapia restrictiva permite alcanzar iguales resultados funcionales, con menor riesgo para el paciente y ahorraría recursos al sistema.

Palabras clave: TRANSFUSIÓN SANGUÍNEA

CIRUGÍA ORTOPÉDICA MAYOR

ANEMIA

Key words: $\quad$ BLOOD TRANSFUSION

MAJOR ORTHOPEDIC SURGERY

ANEMIA

\footnotetext{
* Internista de la Unidad de Cirugía del Aparato Locomotor de la Asociación Española. Ex Asistente de Medicina Interna, Clínica Médica 2, Hospital Pasteur.

† Internista de la Asociación Española. Asistente de Medicina Interna, Clínica Médica 2, Hospital Pasteur.

¥ Médico traumatólogo, residente de la Unidad de Cirugía del Aparato Locomotor de la Asociación Española.

$\S$ Director de la Unidad de Cirugía del Aparato Locomotor, Asociación Española. Ex Prof. Adjunto de Ortopedia y Traumatología.

ๆ Director del Servicio de Medicina Transfusional de la Asociación Española. Ex Prof. de Medicina Transfusional.

** Director del Servicio de Medicina Intensiva y Medicina Interna de la Asociación Española. Ex Prof. de Medicina Intensiva, Hospital de Clínicas.

Los autores declaran no tener conflicto de intereses.

Correspondencia: Dr. Marcos Pintos. Correo electrónico: mepintos@hotmail.com

Recibido: 5/6/17

Aprobado: 25/9/17
} 


\section{Introducción}

Uruguay es el país con mayor proporción de ancianos de Latinoamérica. La artrosis y la fractura de cadera constituyen enfermedades que afectan particularmente a esta población ${ }^{(1,2)}$.

Para pacientes que sufren artrosis avanzada o en algunos casos de fractura de cadera, la solución propuesta es la cirugía ortopédica mayor, entendiéndose por tal la prótesis total de cadera (PTC), la prótesis total de rodilla (PTR) y la prótesis parcial de cadera (PPC) ${ }^{(1-3)}$.

Específicamente en los pacientes que ingresan para cirugía de cadera o rodilla nos enfrentamos a la particularidad de que la mayoría son individuos añosos, con enfermedades crónicas concomitantes, algunos inmunocomprometidos o con otras patologías complejas, por lo que los cuidados a considerar son mayores.

La anemia es un trastorno en el cual el número de glóbulos rojos o su capacidad para el transporte de oxígeno son insuficientes para mantener los requerimientos fisiológicos del organismo. Su diagnóstico en la práctica clínica se realiza a través de las cifras de hemoglobina $(\mathrm{Hb})$ : menor a $13 \mathrm{~g} / \mathrm{dl}$ en hombres, menor a $12 \mathrm{~g} / \mathrm{dl}$ en mujeres y menor a $11 \mathrm{~g} / \mathrm{dl}$ durante el embarazo ${ }^{(4)}$. Puede clasificarse por el tiempo de instalación como aguda o crónica, destacándose que en el primer caso generalmente es peor tolerada (más sintomática) y en el segundo su progresión más lenta permite al organismo poner en marcha mecanismos adaptativos ${ }^{(5,6)}$.

Algunos de estos pacientes requerirán transfusión de sangre o hemocomponenetes, o ambos, antes o después de la intervención. Los riesgos a los que se expone un individuo que recibe sangre alogénica pueden ser múltiples: reacciones inmulógicas, alergia, infecciones, fiebre, edema pulmonar congestivo e injuria pulmonar relacionada a transfusión (TRALI, por su sigla en inglés). La fiebre es la reacción transfusional más frecuente, entendiendo por tal la aparición de fiebre durante o inmediatamente posterior a la administración de sangre, sin evidencia de infección u otro fenómeno sistémico que la explique $^{(6-9)}$.

El abordaje de la anemia en el paciente quirúrgico puede hacerse de manera liberal, sujeto a la indicación de cada cirujano, o en el contexto de un protocolo restrictivo de reposición. En el primer caso la tendencia es a un mayor número de transfusiones.

Hay evidencia que demuestra que la evolución funcional y la sobrevida de los pacientes sometidos a protocolos restrictivos es la misma que los que se someten a prácticas liberales de transfusión. El beneficio en el ahorro de recursos se acompaña además de un menor número de complicaciones relacionadas con la administración de sangre alogénica ${ }^{(7,10-12)}$.
Los criterios de reposición restrictivos se basan en la administración de transfusión de glóbulos rojos solo cuando aparecen síntomas compatibles con síndrome funcional anémico, o cuando los niveles de hemoglobina disminuyen por debajo de $7-8 \mathrm{~g} / \mathrm{dl}$. Serán transfundidos más precozmente aquellos pacientes portadores de enfermedades crónicas como: insuficiencia cardíaca congestiva (ICC), cardiopatía isquémica o insuficiencia renal $^{(6,13)}$.

El objetivo general fue comparar dos estrategias transfusionales: la estrategia liberal y la terapia restrictiva tranfusional (TRT) en los pacientes sometidos a artroplastia de cadera y rodilla.

Para ello, nos planteamos como objetivos específicos: 1) instaurar la TRT; 2) reducir el número de transfusiones; 3) disminuir las complicaciones perioperatorias relacionadas con las transfusiones, y 4) disminuir los costos del servicio.

\section{Material y método}

Este estudio fue realizado en conjunto por los servicios de Medicina Interna, Medicina Transfusional y la Unidad de Cirugía del Aparato Locomotor (UCAL) de la Asociación Española de Montevideo. En este centro se operan aproximadamente 1.000 pacientes por año, y se dedica exclusivamente a la artroplastia de cadera y rodilla.

Metodológicamente fue un estudio comparativo, descriptivo, en base a dos cohortes prospectivas en la que se enrolaron todos los pacientes intervenidos en los trimestres de abril, mayo y junio de los años 2014 y 2015, siendo los primeros sometidos a estrategia liberal y los segundos a TRT.

Los procedimientos más frecuentemente realizados son: PTC, PPC y PTR. La intervención puede ser primaria o recambio.

El esquema de acción comenzó en el posoperatorio de los pacientes intervenidos de artroplastia de cadera y rodilla. Se les realizó seguimiento clínico durante toda la internación y hemograma de control a las 12 y 24 horas posoperatorias, y posteriormente según necesidad.

Todos los pacientes intervenidos en 2014 fueron sometidos a estrategia liberal, entendiendo por tal la reposición hematológica que depende del criterio clínico de cada médico tratante, con un umbral de hemoglobina de $10 \mathrm{~g} / \mathrm{dl}$ para su indicación y con frecuencia (si la cifra de hemoglobina era menor a $9 \mathrm{~g} / \mathrm{dl}$ ) se indicaban dos unidades de glóbulos rojos.

En el año 2015 se aplicó la TRT, en la cual la indicación de transfusión se realizó solo cuando aparecieron síntomas del síndrome funcional anémico, o cuando los niveles de hemoglobina disminuyeron por debajo de 8 $\mathrm{g} / \mathrm{dl}$. Fueron transfundidos más precozmente aquellos 
Tabla 1. Características clínicas de ambos grupos

\begin{tabular}{lccc}
\hline & Estrategia liberal & Estrategia restrictiva & Valor $p$ \\
\hline Sexo & $\mathrm{M}=57 \mathrm{~F}=172$ & $\mathrm{M}=56 \mathrm{~F}=171$ & 0,521 \\
Edad en años & $71,86 \pm 11,12$ & $71,57 \pm 10,07$ & 0,775 \\
\hline Tipo de cirugía & & & 0,213 \\
PTR (artrosis) & 173 & 138 & 50 \\
PTC (artrosis) & 49 & 38 & 11 \\
Cadera por fractura & 32 & & \\
Recambio & 7 & & \\
\hline M: masculino; F: femenino & & & \\
\hline
\end{tabular}

pacientes portadores de enfermedades crónicas como ICC, cardiopatía isquémica o insuficiencia renal.

Como criterio general la indicación de unidades de glóbulos rojos se realizó de a uno, evaluando posteriormente la situación clínica y la hemoglobina del paciente.

Todos los pacientes intervenidos de artroplastia de rodilla y de cadera permanecieron internados en nuestro centro durante siete y tres días, respectivamente. Por tanto el seguimiento prospectivo está referido a ese tiempo de internación.

El equipo médico, las técnicas anestésico quirúrgicas, los implantes colocados y los planes de rehabilitación física fueron constantes en los dos grupos evaluados.

La evolución funcional de los pacientes se evaluó al alta según la tolerancia al ejercicio: aquellos que deambulaban con buena tolerancia, los que se sentaban al borde de cama o los que no alcanzaban ninguno de los dos objetivos.

El análisis estadístico se realizó a través del cálculo de la media y sus medidas de dispersión más la aplicación de $\mathrm{t}$ test (test de Student) para las variables continuas, $\mathrm{y}$ de porcentajes o proporciones y el cálculo de $\mathrm{x}^{2}$ para las variables discretas. Aceptamos como significativo un error $\alpha$ de $5 \%$. Para la correlación entre variables aplicamos el coeficiente de correlación de Pearson. Se utilizó el paquete estadístico SPSS para Windows.

\section{Resultados}

Ingresaron en el estudio 498 pacientes; 261 de estos $(52,4 \%)$ en el año 2014 y por tanto sometidos a terapia liberal, y $237(47,6 \%)$ en el año 2015 manejados con el protocolo de TRT.

Del total de pacientes, $124(24,8 \%)$ eran de sexo masculino y 374 femenino $(75,2 \%$ ) (tabla 1$)$.
La edad media de presentación fue de 71,7 años, con un rango de 19 a 98 años. Separados por año de cirugía: $71,86 \pm 11,12$ en 2014 y 71,57 $\pm 10,07$ en 2015 ( $\mathrm{p}=$ $0,775)$ (tabla 1).

La intervención quirúrgica más frecuente fue la PTR $(62,4 \%)$ seguida por la PTC por artrosis $(19,9 \%)$ y en tercer lugar la artroplastia de cadera por fractura $(13,9 \%)$. Con menos frecuencia recambios de cadera y rodilla. No hubo diferencias significativas en el número y tipo de procedimientos que se realizaron en ambos grupos (tabla 1).

Se transfundieron en total 99 individuos, 64 en 2014 y 35 en 2015 con un valor $p=0,002$ (tabla 2).

Se utilizaron en total 210 unidades de glóbulos rojos, 144 en 2014 y 66 en 2015 ( $p=0,0001)$.

La intervención que requirió más transfusiones fue la artroplastia de cadera por fractura, alcanzando casi una de cada tres unidades utilizadas en el período restrictivo.

Las cifras medias de hemoglobina al alta fueron de 10,55 y 10,25 en 2014 y 2015 respectivamente $(p=0,49)$.

Se presentaron diez casos de reacción transfusional, todas de tipo febril. De estas, ocho se constataron en 2014 y dos en $2015(\mathrm{p}=0,073)$.

Vimos que existe una relación estadísticamente significativa entre la necesidad de transfusión y la situación al alta $(\mathrm{p}=0,001)$, con un coeficiente de correlación negativo, siendo peor la clase funcional en relación con la necesidad de transfusión $(\mathrm{p}=0,001)$.

Cuando observamos la evolución funcional de los pacientes en función del año de la intervención obtuvimos los datos expuestos en la tabla 3 , con un valor $\mathrm{p}=$ 0,53 . 
Tabla 2. Cifras comparativas entre grupos

\begin{tabular}{lccc}
\hline Estrategia & Liberal & Restrictiva & Valor $p$ \\
\hline Unidades de glóbulos rojos & 144 & 66 & 0,0001 \\
Pacientes transfundidos & 64 & 35 & 0,002 \\
Hb al alta g/dl & 10,55 & 10,25 & 0,49 \\
Reacciones transfusionales & 8 & 2 & 0,073 \\
\hline
\end{tabular}

\section{Discusión y conclusiones}

Al aplicar la TRT disminuyó el número de transfusiones realizadas. Se transfundieron aproximadamente la mitad de los pacientes con una diferencia estadísticamente significativa. Al mismo tiempo se utilizaron $55 \%$ menos de unidades de glóbulos rojos, lo que muestra un ahorro del recurso significativo. El resultado es similar a lo obtenido por otros servicios de cirugía ortopédica cuando se aplicó la terapia restrictiva ${ }^{(7,13)}$.

La intervención que requirió más transfusiones fue la prótesis de cadera por fractura. Es bien sabido que los pacientes con fractura de cadera son los más vulnerables, con más comorbilidades, y que tienden a presentar más complicaciones. En este grupo de pacientes algunos autores no encontraron diferencias en la morbimortalidad comparando ambas estrategias, pero sí cuando se selecciona la población más frágil y de mayor edad, siendo mayor en los sometidos a TRT ${ }^{(12,14)}$. En el año 2015 se publicó un ensayo clínico controlado (FOCUS trial) que incluyó a pacientes provenientes de 47 hospitales de Estados Unidos y Canadá, todos mayores de 50 años y con factores de riesgo o enfermedad cardiovascular declarada que se sometieron a cirugía por fractura de cadera y demostraron que la estrategia restrictiva no afecta la mortalidad con referencia a la estrategia libe$\mathrm{ral}^{(11)}$. Estos estudios clínicos realizan un seguimiento de la población estudiada de 30 a 90 días, lo que escapa a los objetivos de nuestra investigación, pero abonan el concepto de que en los pacientes más añosos y vulnerables hay que ser cuidadoso al momento de evaluar la indicación de transfusión.

De capital importancia es la disminución de reacciones adversas transfusionales. Estas pueden tener múltiples presentaciones y con diferente gravedad, e incluso pueden llevar a la muerte. En la población estudiada comprobamos ocho casos de reacción febril con la estrategia liberal y dos con la restrictiva. Observando así una clara tendencia estadística (si bien no obtuvimos una diferencia significativa), para lo que seguramente necesitaríamos un mayor número de pacientes. La mayoría de las revisiones y metaanálisis se basan en ensayos clíni-
Tabla 3. Situación funcional al alta versus estrategia utilizada

\begin{tabular}{lccc}
\hline Estrategia & Decúbito & Se sienta & Deambula \\
\hline Liberal & 4 & 24 & 197 \\
Restrictiva & 2 & 21 & 214 \\
Total & 6 & 45 & 411 \\
\hline
\end{tabular}

cos heterogéneos en los que se analizan eventos como mortalidad, infarto de miocardio, falla cardíaca, infecciones y clase funcional, sin considerar las reacciones transfusionales propiamente dichas, pero es sensato pensar que estas disminuyen en la medida que disminuyen las transfusiones ${ }^{(6-8,10,11)}$.

Con respecto a la evolución funcional no se comprobó diferencia entre los operados que se sometieron a la estrategia liberal frente a la restrictiva, lo que confirma los hallazgos obtenidos en experiencias previas. Está claro que la situación clínica del paciente, más que las cifras de hemoglobina, es el elemento más importante a evaluar para la indicación de transfusión ${ }^{(5-7,7,12,13)}$.

Mao T y colaboradores publicaron en 2017 una revisión sistemática y metaanálisis que concluye que la TRT no tiene ventajas sobre la estrategia liberal aunque claramente se transfunden menos pacientes ${ }^{(10)}$.

Recientemente, la revista JAMA publicó una guía de práctica clínica basada en evidencia que recomienda la TRT y propone la cifra de $8 \mathrm{~g} / \mathrm{dl}$ de hemoglobina como umbral para la transfusión en los pacientes intervenidos de $\operatorname{artroplastia}^{(15)}$.

En nuestra experiencia y de acuerdo a la literatura consultada la terapia restrictiva permite alcanzar iguales resultados funcionales, con menor riesgo para el paciente y ahorraría recursos al sistema.

\section{Summary}

Major orthopedic surgery is the surgical solution proposed for patients who suffer from advanced arthrosis or for some cases of hip fracture.

Some of these patients will require blood and/or blood components transfusion before or after surgery. The approach to anemia in surgical patients may involve a liberal or a restrictive transfusion strategy, according to what each surgeon indicates, or observing the context of a restrictive protocol for blood replacement. The latter results in resource savings and in a reduction in the number of complications due to the administration of allogenic blood.

The study aims to compare the liberal transfusion strategy to the restrictive strategy. 
498 patients were included in the study, 261 of which $(52.4 \%)$ were treated within the liberal strategy in 2014, and 237 (47.6\%) were treated according to a restrictive transfusion protocol in 2015.

A smaller number of individuals received blood transfusions and 55\% less blood volumes were needed when the restrictive strategy was applied.

Ten cases of transfusion reaction arose, all of them involving fever, 8 in 2014 and 2 in 2015.

As to the functional evolution, no differences were found between the two groups.

According to our study, and according to the global literature consulted, the restrictive therapy strategy enables the same functional results with a lower risk for patients, and it saves resources for the system.

\section{Bibliografía}

1. Fondo Nacional de Recursos. Artroplastia de cadera por artrosis. Montevideo: FNR, 2010.

2. Fondo Nacional de Recursos. Artroplastia de cadera por frasctura. Montevideo: FNR, 2010.

3. Fondo Nacional de Recursos. Artroplastia de rodilla. Montevideo: FNR, 2010.

4. Organización Mundial de la Salud. Concentraciones de hemoglobina para diagnosticar la anemia y evaluar su gravedad. Ginebra: OMS, 2011. Disponible en: http://www.who.int/ vmnis/indicators/haemoglobin_es.pdf. [Consulta: ].

5. Kassebaum NJ; GBD 2013 Anemia Collaborators. The Global Burden of Anemia. Hematol Oncol Clin North Am 2016; 30(2):247-308.

6. Madrazo González, Z, García Barrasa A, Rodríguez Lorenzo L, Rafecas Renau A, Alonso Fernández G. Actualización en anemia y terapia transfusional. Med Intensiva 2011; 35(1):32-40.
7. Carson JL, Terrin ML, Noveck H, Sanders DW, Chaitman BR, Rhoads GG, et al. Liberal or restrictive transfusion in high-risk patients after hip surgery. N Engl J Med 2011; 365(26):2453-62.

8. Toy P, Popovsky MA, Abraham E, Ambruso DR, Holness LG, Kopko PM, et al; National Heart, Lung and Blood Institute Working Group on TRALI. Transfusion-related acute lung injury: definition and review. Crit Care Med 2005; 33(4):721-6.

9. Organización Mundial de la Salud. El uso clìnico de la sangre: manual de bolsillo. Ginebra: OMS, 2001.

10. Mao T, Gao F, Han J, Sun W, Guo W, Li Z, et al. Restrictive versus liberal transfusion strategies for red blood cell transfusion after hip or knee surgery: a systematic review and meta-analysis. Medicine (Baltimore) 2017; 96(25):e7326.

11. Carson JL, Sieber F, Cook DR, Hoover DR, Noveck H, Chaitman BR, et al. Liberal versus restrictive blood transfusion strategy: 3-year survival and cause of death results from the FOCUS randomised controlled trial. Lancet 2015; 385(9974):1183-9.

12. Brunskill SJ, Millette SL, Shokoohi A, Pulford EC, Doree C, Murphy MF, et al. Red blood cell transfusion for people undergoing hip fracture surgery. Cochrane Database Syst Rev 2015; (4):CD009699.

13. García García E. Pautas transfusionales en el paciente quirúrgico. Rev Cuba Anestesiol Reanim 2013; 12(3):231-43.

14. Gregersen M, Borris LC, Damsgaard EM. Postoperative blood transfusion strategy in frail, anemic elderly patients with hip fracture: the TRIFE randomized controlled trial. Acta Orthop 2015; 86(3):363-72.

15. Carson JL, Guyatt G, Heddle NM, Grossman BJ, Cohn CS, Fung MK, et al. Clinical practice guidelines from the AABB: red blood cell transfusion thresholds and storage. JAMA 2016; 316(19):2025-2035. 